\title{
New Developments in Vector Velocity Imaging using the Transverse Oscillation Approach
}

Jensen, Jørgen Arendt; Pihl, Michael Johannes; Olesen, Jacob Bjerring; Møller Hansen, Peter; Lindskov Hansen, Kristoffer; Bachmann Nielsen, Michael

\author{
Published in: \\ Proceedings of SPIE \\ Link to article, DOI: \\ $10.1117 / 12.2013701$ \\ Publication date: \\ 2013
}

Link back to DTU Orbit

Citation (APA):

Jensen, J. A., Pihl, M. J., Olesen, J. B., Møller Hansen, P., Lindskov Hansen, K., \& Bachmann Nielsen, M. (2013). New Developments in Vector Velocity Imaging using the Transverse Oscillation Approach. In Proceedings of SPIE: Medical Imaging 2013: Ultrasonic Imaging, Tomography, and Therapy (Vol. 8675, pp. 86750F). SPIE - International Society for Optical Engineering. https://doi.org/10.1117/12.2013701

\section{General rights}

Copyright and moral rights for the publications made accessible in the public portal are retained by the authors and/or other copyright owners and it is a condition of accessing publications that users recognise and abide by the legal requirements associated with these rights.

- Users may download and print one copy of any publication from the public portal for the purpose of private study or research.

- You may not further distribute the material or use it for any profit-making activity or commercial gain

- You may freely distribute the URL identifying the publication in the public portal 


\title{
New Developments in Vector Velocity Imaging using the Transverse Oscillation Approach
}

\author{
Jørgen Arendt Jensen ${ }^{a}$, Michael Johannes Pihl ${ }^{a}$, Jacob Bjerring Olesen ${ }^{a}$, \\ Peter Møller Hansen ${ }^{b}$, Kristoffer Lindskov Hansen ${ }^{b}$ and Michael Bachmann Nielsen ${ }^{b}$ \\ ${ }^{a}$ Center for Fast Ultrasound Imaging, Department of Electrical Engineering, \\ Technical University of Denmark, DK-2800 Lyngby, Denmark; \\ ${ }^{b}$ Department of Radiology, Rigshospitalet, Copenhagen University Hospital, Denmark
}

\begin{abstract}
Vector velocity imaging using the Transverse Oscillation (TO) approach has recently been FDA approved for linear array transducers on a commercial platform. It can now be used clinically for studying the complex flow at e.g. bifurcations, valves, and the heart in real time. Several clinical examples from venous flow to rotational flow in the heart will be shown. The technique is also being further developed and adapted for convex and phased array probes, for spectral velocity estimation, pressure estimation, and for three dimensional velocity tensor imaging. It is shown how the methods are optimized using Field II simulations along with several examples of their performance.
\end{abstract}

Keywords: Medical Ultrasound, Vector velocity imaging, Transverse oscillation

\section{INTRODUCTION}

The blood flow in the human circulation is highly pulsatile and evolves in short vessels with numerous branches, bifurcations, bends, and geometric changes. This results in highly complex flow patterns in both normal and diseased vessels and across venous and cardiac valves. The flow is turbulent at parts of the cardiac cycle and vortices are formed. A complete visualization and characterization of this complex flow necessitates a vector velocity measurement system with a high temporal resolution.

Current medical ultrasound systems only measure the axial velocity component along the ultrasound beam and must therefore angle correct the results to obtain quantitative measurements. An alternative is to use MR scanners, but these are restricted to using ECG gating and measurements over many cardiac cycles (often several hundred) to piece together a three-dimensional measurement of the blood flow. ${ }^{1,2}$ This is inconvenient to the patient, expensive and can introduce gross artifacts for irregular heart rhythms.

Recently a new method for ultrasound vector velocity estimation has been FDA approved and introduced on the market. ${ }^{3,4}$ The method employs a double oscillating ultrasound field and dedicated estimators for finding both the axial and lateral velocity components of the instantaneous velocity. The system can visualize the complex velocity field showing 20 to 60 frames per second in real time for revealing the hemodynamics in the body. This paper presents the basic methodology for making the estimation in the current implementation for a linear array probe. It is also explained how the double oscillating fields can be optimized to reduce bias and increase accuracy of the estimates. A range of new possibilities are being made available with this approach. This includes expanding the method for new probe geometries like the phased array for cardiac scanning and convex arrays for abdominal scanning. It is also possible to expand the method for full three-dimensional scanning, so that all three velocity components are estimated simultaneously from the same data using a two-dimensional array for three-dimensional volumetric velocity tensor imaging in real time.

New measurements are also made possible by combining the approach with new estimation schemes as described in Section 6. This includes finding the velocity distribution at any angle using spectral velocity estimation based on the TO ultrasound field. Another interesting area is to find pressure gradients by solving the Navier-Stokes equation based on the estimated velocity field.

Further author information: Send correspondence to J. A. Jensen, E-mail: jaj@elektro.dtu.dk 


\begin{tabular}{|l|c|}
\hline Transducer center frequency $f_{0}$ & $3.4 \mathrm{MHz}$ \\
Number of cycles in emitted pulse & 8 \\
Wavelength & $0.45 \mathrm{~mm}$ \\
Element pitch & $0.33 \mathrm{~mm}$ \\
Kerf & $16.5 \mu \mathrm{m}$ \\
Height of element & $13 \mathrm{~mm}$ \\
Fixed elevation focus & $90 \mathrm{~mm}$ \\
Number of transducer elements & 192 \\
Convex radius & $60 \mathrm{~mm}$ \\
Electronic focus in transmit & $100 \mathrm{~mm}$ \\
Depth for optimizing the transmit focusing & $40 \mathrm{~mm}$ \\
F\# in transmit & 2 \\
Number of receive elements & 128 \\
Width of apodization peaks & 64 \\
Apodization function & Hamming \\
\hline
\end{tabular}

Table 1. Standard set-up for generating the transverse oscillating field for a convex array probe.

The paper is concluded by showing a number of clinical examples from use of the FDA approved scanner in Section 7. This shows how angle independent measurements are attained and several examples of complex flow in bifurcations, across venous valves, and in the heart are given. A new and interesting application for characterizing the flow during cardiac surgery is demonstrated.

\section{VECTOR VELOCITY ESTIMATION USING TRANSVERSE OSCILLATION}

The blood velocity can be estimated in medical ultrasound by finding the shift in position of the scatterers between consecutive pulse emissions. ${ }^{5,6}$ This is often performed by finding the phase shift between two consecutive received signals using a phase shift estimator. ${ }^{7,8}$ The key element here is that a sinusoidal oscillating pulse is found in the axial direction and a phase shift between received signals can therefore be determined from this. Complex received data must be employed to determine the sign of the velocity, so the received RF signals are Hilbert transformed to create the analytic signal, where the quadrature signal is $90^{\circ}$ phase shifted compared to the received in-phase RF signal.

The basic idea in the transverse oscillation (TO) approach is to create a sinusoidal oscillation transverse to the ultrasound propagation direction and thereby make it possible to estimate the transverse velocity component. ${ }^{9-11}$ The lateral motion's direction is determined from the lateral analytic signal. Therefore two receive fields are synthesized from a single pulse emissions to create a quadrature lateral signal, where the left and right fields are phase shifted $90^{\circ}$ compared to each other. Two estimators are then used for finding the axial and transverse velocity components independently, but at the same time from the same data. ${ }^{12}$

The lateral field at the focus of an ultrasound transducer can, for a monochromatic excitation, be approximated by the Fourier transform of the apodization function. ${ }^{13}$ The apodization function should therefore contain two peaks to create a sinusoidal oscillation in the lateral direction. This can be made with modern multi-element probes and the transverse oscillation is created by having a receive apodization function containing two distinct peaks. ${ }^{10}$ The separation between the peaks determines the lateral oscillation period $\lambda_{x}$ given by

$$
\lambda_{x}=\frac{2 \lambda D}{P_{d}}
$$

where $\lambda=c / f_{0}$ is the axial wave length, $c$ the speed of sound, $f_{0}$ the transducer center frequency, $D$ is the depth and $P_{d}$ is the distance between the two peaks in the apodization function. The ratio $D / P_{d}$ can also be seen as the $F \#$ for the system. The lateral wavelength is influenced by the transmit field, the focusing in transmit and receive, and the excitation pulse used. The exact prediction of the field therefore has to be made using simulations, and Field II is used in this paper for calculating the field. ${ }^{14,15}$ 

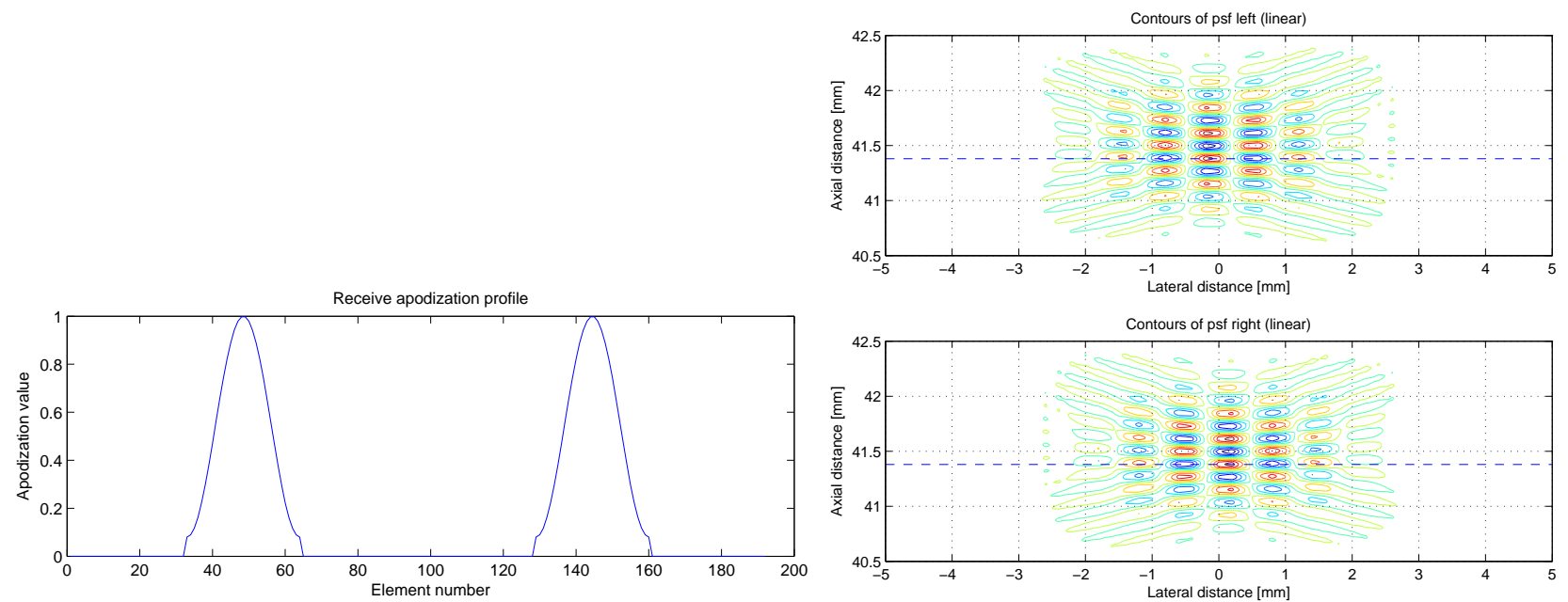

Figure 1. The left graph shows the receive apodization profile as a function of element number. Linear contour plots of the left and right point spread functions at a depth of $40 \mathrm{~mm}$ are shown at the right graphs. Note how the two fields are laterally shifted 90 degrees compared to each other at the center.
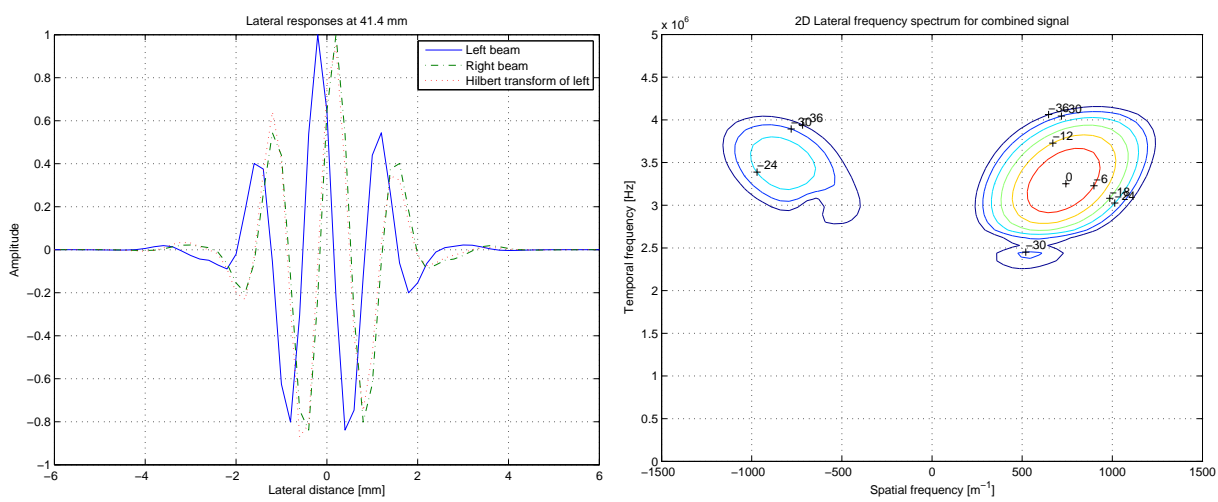

Figure 2. The left graph shows the lateral oscillation field for the left and right PSF around the peak value in the fields. The right graph shows the amplitude of the two-dimensional Fourier transform of the complex transverse oscillation field with $6 \mathrm{~dB}$ between contours.

The parameters for such a setup are shown in Table 1 for a commercial convex array probe. The apodization function employed is shown in Fig. 1 with a separation between the peaks of 64 elements and using 64 active elements in receive. The figure also shows the resulting left and right point spread functions in a linear contour plot. The fields are slightly displaced to either the left or right compared to the center line. The horizontal lines show the depth (time) for taking out the lateral response shown in Fig. 2. The two lateral signals nearly form a Hilbert transform pair, where the right response is the Hilbert transform of the left signal. The dotted line indicates the signal obtained from performing a Hilbert transform of the left signal, and it can be seen that periodicity and main phase shift is close to the correct Hilbert transform, but there are slight variations in amplitude. This results in spectral leakage from positive frequencies to negative frequencies as shown in the right plot in Fig. 2. It shows the two-dimensional Fourier transform of the complex transverse oscillation field, where the left field is the real part and the right field is the imaginary part. Ideally there should be no energy for negative spatial frequencies, but a peak at negative frequencies are found due to the non-ideal Hilbert relation. The $\lambda_{x}$ found by (1) is often optimistic and too low, and the spacing between the in-phase and quadrature beams is hereby to small. The focusing does therefore not result in a proper Hilbert transform pair and the interplay between $\lambda_{x}$, the apodization profile, and the focusing must be simulated to optimize the spatial quadrature field. 


\section{VELOCITY ESTIMATION}

The data beamformed for the approach contains a mixture of the axial and lateral velocities, which cannot be separated by conventional velocity estimators. There is, thus, a need for two dedicated estimators for each of the velocity components. The data acquisition yields two beams focused in parallel, where the left one contains the in-phase and the right the quadrature component. This complex signal can, at one fixed depth, be described by: ${ }^{12}$

$$
r_{s q}(i)=\cos \left(2 \pi f_{p} i T_{p r f}\right) \exp \left(j 2 \pi f_{x} i T_{p r f}\right)
$$

where $i$ is the emission number, $T_{p r f}$ is the pulse repetition time, $f_{x}=1 / \lambda_{x}$, and $f_{p}$ is the received axial frequency given by:

$$
f_{p}=\frac{2 v_{z}}{c} f_{0}
$$

Here $v_{z}$ is the axial velocity component. The temporal Hilbert transform of (2) is

$$
r_{s q h}(i)=\sin \left(2 \pi f_{p} i T_{p r f}\right) \exp \left(j 2 \pi f_{x} i T_{p r f}\right) .
$$

Combining (2) and (4) and using Euler's equations gives

$$
\begin{aligned}
r_{s q}(i) & =\frac{1}{2}\left(\exp \left(j 2 \pi i T_{p r f}\left(f_{x}+f_{p}\right)\right)+\exp \left(j 2 \pi i T_{p r f}\left(f_{x}-f_{p}\right)\right)\right) \\
r_{s q h}(i) & =\frac{1}{2 j}\left(\exp \left(j 2 \pi i T_{p r f}\left(f_{x}+f_{p}\right)\right)-\exp \left(j 2 \pi i T_{p r f}\left(f_{x}-f_{p}\right)\right)\right) .
\end{aligned}
$$

Two new signals are then formed:

$$
r_{1}(i)=r_{s q}(i)+j r_{s q h}(i)=\exp \left(j 2 \pi i T_{p r f}\left(f_{x}+f_{p}\right)\right), \quad r_{2}(i)=r_{s q}(i)-j r_{s q h}(i)=\exp \left(j 2 \pi i T_{p r f}\left(f_{x}-f_{p}\right)\right) .
$$

The autocorrelation functions of $r_{1}(i)$ and $r_{2}(i)$ are found as

$$
R_{1}(k)=\sum_{i=1}^{M} r_{1}(i) r_{1}(i+k)
$$

where $M$ is the number of emissions. The velocity components can then be estimated independently by two estimators given by: ${ }^{12}$

$$
v_{x}=\frac{\lambda_{x}}{2 \pi 2 T_{\text {prf }}} \arctan \left(\frac{\Im\left\{R_{1}(1)\right\} \Re\left\{R_{2}(1)\right\}+\Im\left\{R_{2}(1)\right\} \Re\left\{R_{1}(1)\right\}}{\Re\left\{R_{1}(1)\right\} \Re\left\{R_{2}(1)\right\}-\Im\left\{R_{1}(1)\right\} \Im\left\{R_{2}(1)\right\}}\right)
$$

and

$$
v_{z}=\frac{c}{2 \pi 4 T_{p r f} f_{0}} \arctan \left(\frac{\Im\left\{R_{1}(1)\right\} \Re\left\{R_{2}(1)\right\}-\Im\left\{R_{2}(1)\right\} \Re\left\{R_{1}(1)\right\}}{\Re\left\{R_{1}(1)\right\} \Re\left\{R_{2}(1)\right\}+\Im\left\{R_{1}(1)\right\} \Im\left\{R_{2}(1)\right\}}\right) .
$$

where $\Im\left\{R_{1}(1)\right\}$ denotes the imaginary part of the lag one autocorrelation and $\Re\left\{R_{1}(1)\right\}$ the corresponding real part. So from the same acquired data the two velocity components can be estimated simultaneously.

\section{EVALUATION OF TRANSVERSE OSCILLATION FIELDS}

The estimation result from the method is directly linked to the field generated and (1) only gives a rough estimate of $\lambda_{x}$. Simulation of the pulsed field must be employed to accurately determine $\lambda_{x}$ and to optimize the field for velocity estimation. The complex field can be characterized by its estimated spatial mean frequency given by:

$$
\bar{f}_{\text {space }}=\frac{\int_{-f_{s} / 2}^{+f_{s} / 2} \int_{-f_{s x} / 2}^{+f_{s x} / 2} f_{\text {space }}\left|H\left(f_{\text {time }}, f_{\text {space }}\right)\right|^{2} d f_{\text {time }} d f_{\text {space }}}{\int_{-f_{s} / 2}^{+f_{s} / 2} \int_{-f_{s x} / 2}^{+f_{s x} / 2}\left|H\left(f_{\text {time }}, f_{\text {space }}\right)\right|^{2} d f_{\text {time }} d f_{\text {space }}},
$$


where $H\left(f_{\text {time }}, f_{\text {space }}\right)$ is the Fourier transform of the point spread function. This directly scales the velocity estimate as $\lambda_{x}=1 / \bar{f}_{\text {space }}$ in (8). A precise knowledge of the lateral oscillation period is, thus, needed and can be estimated by (10). The lateral oscillation period also affects the receive beamformation as the left and right beam have to be shifted a quarter wavelength compared to each other.

A measure of the spectral spread is obtained by:

$$
\sigma_{\bar{f}_{\text {space }}}^{2}=\frac{\int_{-f_{s} / 2}^{+f_{s} / 2} \int_{-f_{s x} / 2}^{+f_{s x} / 2}\left(f_{\text {space }}-\bar{f}_{\text {space }}\right)^{2}\left|H\left(f_{\text {time }}, f_{\text {space }}\right)\right|^{2} d f_{\text {time }} d f_{\text {space }}}{\int_{-f_{s} / 2}^{+f_{s} / 2} \int_{-f_{s x} / 2}^{+f_{s x} / 2}\left|H\left(f_{\text {time }}, f_{\text {space }}\right)\right|^{2} d f_{\text {time }} d f_{\text {space }}} .
$$

This can form the basis for the optimization of the spatial quadrature field and minimizing $\sigma_{\bar{f}_{\text {space }}}^{2}$ will give the best result in terms of the most narrow spectrum as this directly affects the variance of the estimated lateral velocity. Another parameter to optimize is to reduce the spectral leakage ratio $L_{r}$, so that most of the energy is concentrated for positive spatial frequencies. This is calculated as

$$
L_{r}=\frac{\int_{-f_{s} / 2}^{+f_{s} / 2} \int_{-f_{s x} / 2}^{0}\left|H\left(f_{\text {time }}, f_{\text {space }}\right)\right|^{2} d f_{\text {time }} d f_{\text {space }}}{\int_{-f_{s} / 2}^{+f_{s} / 2} \int_{-f_{s x} / 2}^{+f_{s x} / 2}\left|H\left(f_{\text {time }}, f_{\text {space }}\right)\right|^{2} d f_{\text {time }} d f_{\text {space }}} .
$$

A high leakage will bias the estimate downwards as the mean frequency of the field is found by the phase shift estimator. ${ }^{6,7}$

For the field shown in the previous figures the performance indicators are calculated to be: Lateral oscillation frequency $\bar{f}_{\text {space }}=705.5 \mathrm{~m}^{-1}$ and the wavelength is $1.42 \mathrm{~mm}$. The value calculated by (1) is $1.14 \mathrm{~mm}$. The spectral leakage ratio is $L_{r}=1.99 \%$ equivalent to $-17.0 \mathrm{~dB}$ and the standard deviation of the spectrum is $\sigma_{\bar{f}_{\text {space }}}=124.9 \mathrm{~m}^{-1}$.

\section{OPTIMIZATION OF THE LATERAL OSCILLATION FIELD}

The lateral oscillating field can be optimized by changing the focusing, the apodization, the number of elements, the transmitted field, and the number of oscillations in the emitted pulse. This will be made in this Section.

The lateral oscillation period is estimated from (1), but often this prediction is lower than what is actually attained. Displacing the in-phase and quadrature fields $\lambda_{x} / 8$ to either side of the center of the emission does therefore not give two beams $90^{\circ}$ phase shifted compared to each other. A variation of the assumed lateral oscillation period and the resulting parameters are shown in the left graphs in Fig. 3, where the top graph shows the obtained mean frequency as a function of the wavelength sought achieved during the receive focusing. The middle graph shows the standard deviation of the field, and a low value is beneficial as this will give the lowest variance on the estimate. The bottom graph shows the spectral leakage ratio and a low ratio will give a reduced bias in the estimate. The best value for $\lambda_{x}$ in all graphs lies above the estimated lateral wavelength of $1.14 \mathrm{~mm}$.

In the right graph in Fig. 3 the estimated lateral wavelength found from (10) is shown as a function of the assumed wavelength used for designing the focusing. It can be seen that setting the wavelength to $\lambda_{x}=1.37 \mathrm{~mm}$ gives a field, where the desired and obtained wavelength are the same, and this also roughly corresponds to the optimal value given in the right graphs in Fig. 3. After optimization the mean lateral oscillations frequency is: $725.3 \mathrm{~m}^{-1}$ and the wavelength is $1.38 \mathrm{~mm}$. The fractional energy below zero frequency has been reduced to -23.5 $\mathrm{dB}$ and the std. of the lateral oscillations frequency is now $121.8 \mathrm{~m}^{-1}$. The optimized field can be translated to a lower bias and standard deviation in the final velocity estimates as demonstrated by Pihl et al. ${ }^{16}$ The optimized field is compared to the previous field in Fig. 4. 

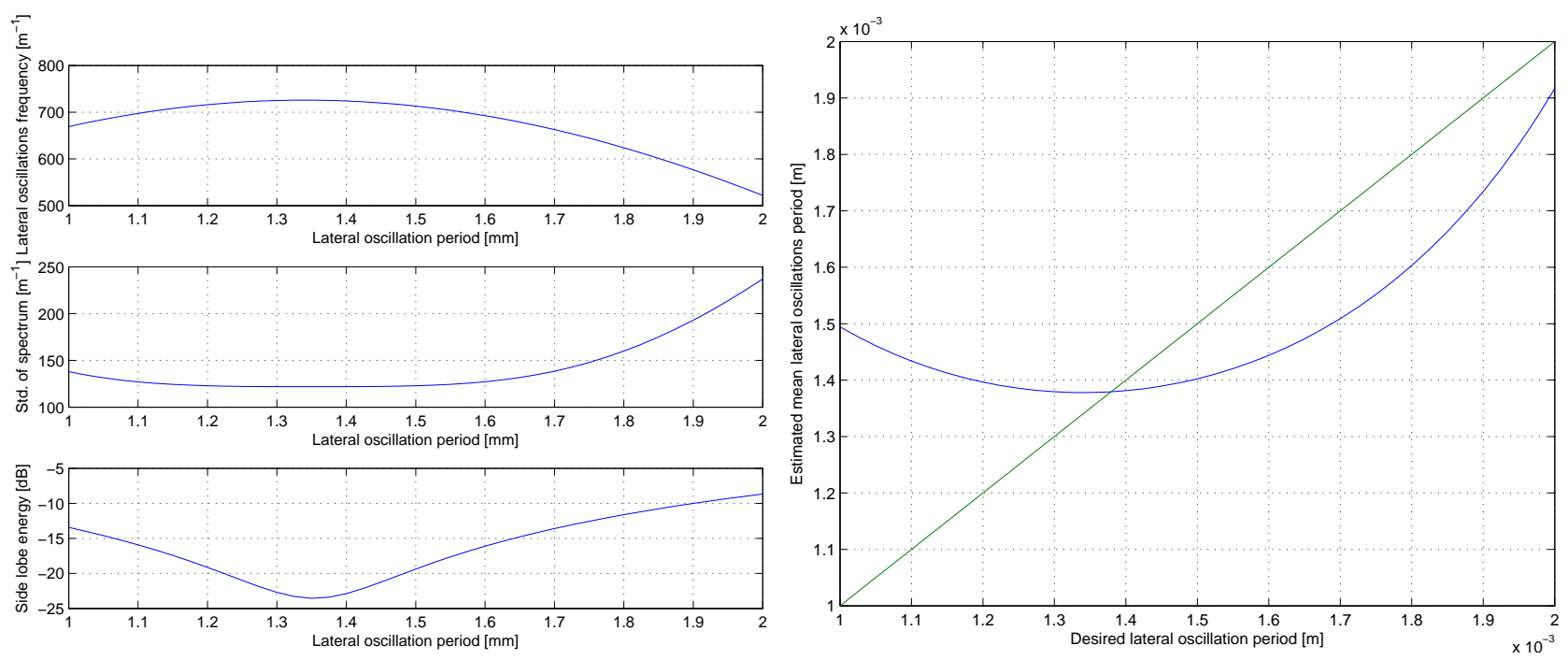

Figure 3. Influence of a variation in the desired lateral oscillation period is shown in the left graphs. The top graph shows the obtained mean frequency, the middle graph the standard deviation of the field, and the lower graph the spectral leakage ratio. The right graph shows the obtained mean lateral oscillation period as a function of the desired period in the blue curve. The green curve is the ideal solution.
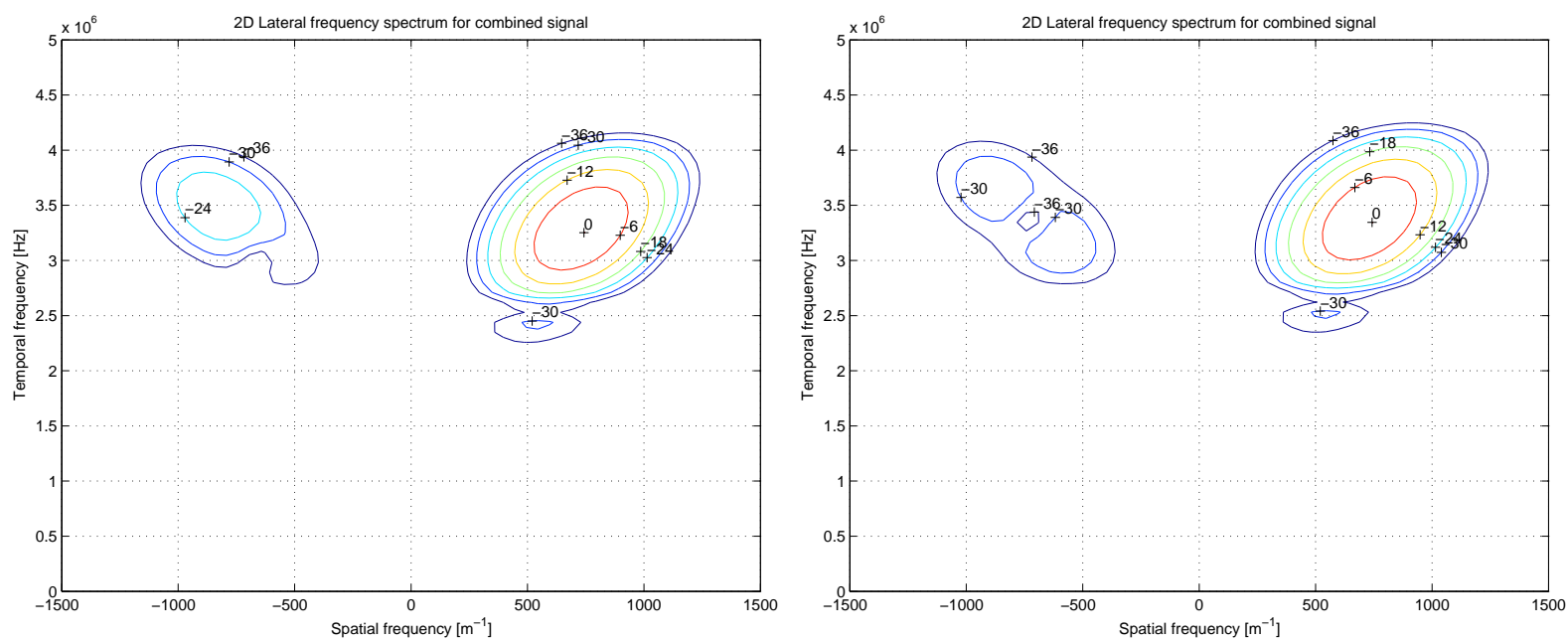

Figure 4. Two-dimensional Fourier transform of the original (left) and optimized (right) complex transverse oscillation field. 

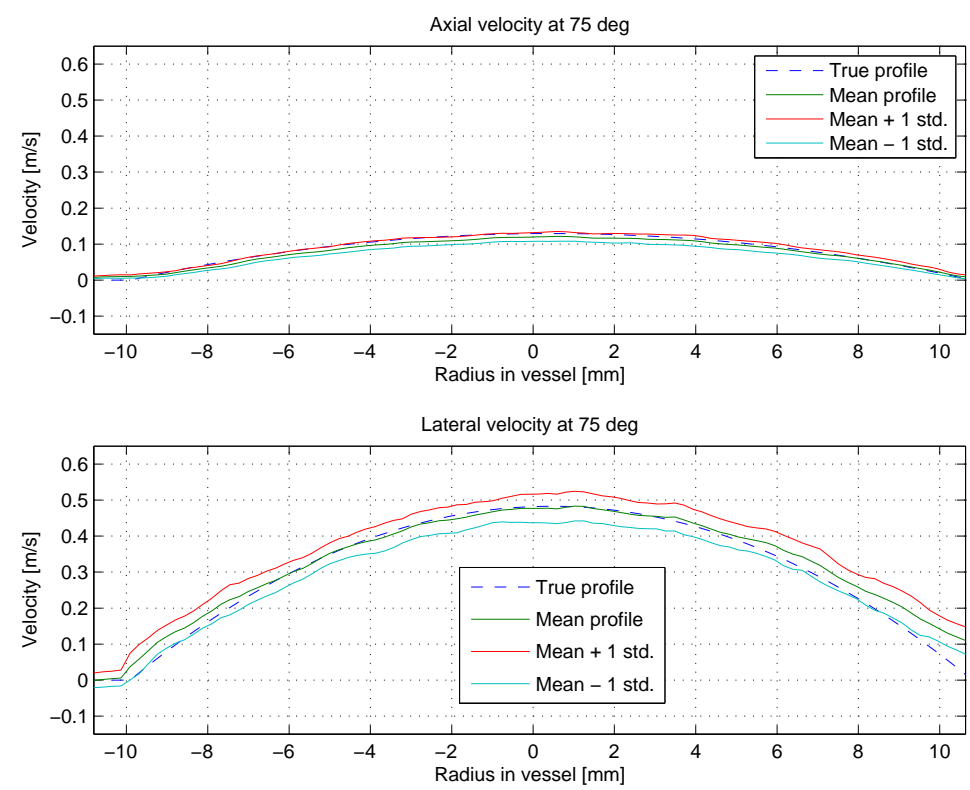

Figure 5. Velocity profiles obtained at a beam-to-flow angle of 75 degrees at a vessel depth of 40 mm for a convex array.

\subsection{Convex array TO}

The current implementation of TO vector flow imaging has been made for linear array transducers. ${ }^{17,18}$ The setup described in the previous Section was optimized for a convex array probe using 64 elements in transmit and 128 in receive with transmit focusing at $100 \mathrm{~mm}$ and dynamic receive focusing using a two peaks apodization profile. This setup can be used for making TO vector velocity imaging with a convex array.

Parabolic velocity profiles have been simulated using Field II for 2000 pulse-echo lines and a vessel at a depth of $40 \mathrm{~mm}$. Typical velocity profiles are shown in Fig. 5 for a beam-to-flow angle of 75 degrees. The axial velocity is shown on the top graph and the lateral component on the bottom. The ideal profile is represented by the dashed line, the green line is the mean of the 125 estimates and the span is \pm one standard deviation. Sixteen emissions are used for finding each velocity estimate and the mean relative standard deviation for the transverse velocity is $7.3 \%$ and $2.1 \%$ for the axial velocity compared to the peak velocity of $0.5 \mathrm{~m} / \mathrm{s}$ demonstrating that convex array vector flow imaging is possible for optimized TO fields.

\subsection{Phased array TO}

An important area for vector velocity estimation is cardiac imaging, where probing between the ribs is performed. This necessitates the use of phased array probes and the TO approach has recently been adapted to work with these. ${ }^{16,19-21}$ Here the beamforming is made to produce a fixed angle between the in-phase and quadrature beam as shown in Fig. 6 . The angle is given by: ${ }^{16}$

$$
\theta_{T O}=2 \arctan \frac{\lambda_{x} / 8}{z_{0}}=2 \arctan \frac{\lambda_{z}}{4 d_{e}},
$$

where $d_{e}$ is the effective distance between the TO peaks in the apodization function calculated as $d_{e}=d \cos \theta_{0}$, where $\theta_{0}$ is the steering angle for the imaging direction. The phased array beams diverge with a fixed angle instead of beamforming the TO lines with a fixed lateral distance as in linear array imaging.

The approach has been implemented on both the experimental SARUS scanner ${ }^{22}$ and a BK Medical ProFocus scanner equipped with a research interface. ${ }^{23}$ Data were acquired from a flow rig with a parabolic, stationary flow using a $3 \mathrm{MHz}, 64$ elements phased array probe. ${ }^{16,24}$ The resulting profiles and images are shown in Fig. 7, where the vessel is placed at depths of 72 and $82 \mathrm{~mm}$. The top graphs show the individual profiles estimated 


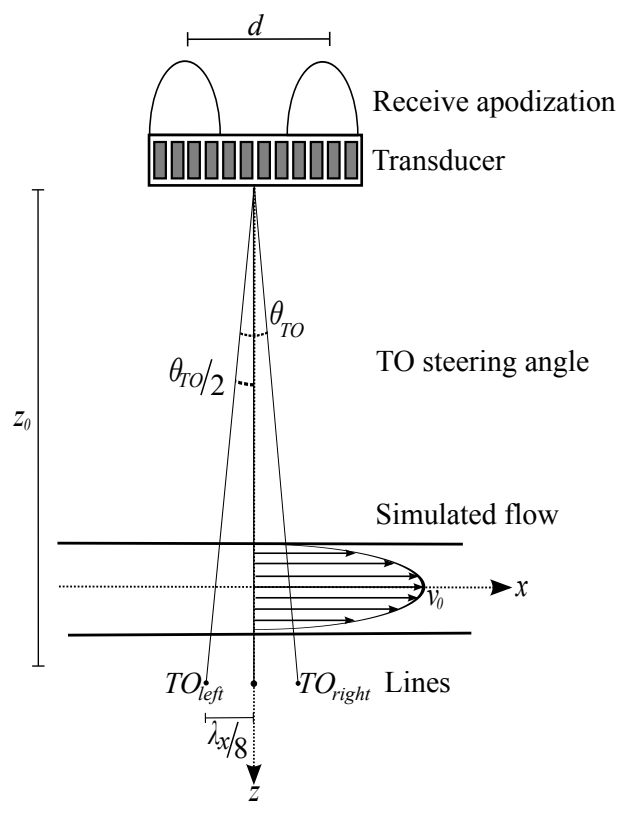

Figure 6. Beamforming approach based on a fixed receive apodization function with a fixed angle between beamformed lines. This angle corresponds to the increase of the spatial lateral wavelength with depth (from Pihl et al. ${ }^{16}$ ).

(left) and the mean \pm one std. (right). The two images below show the resulting vector velocity images at 72 and $82 \mathrm{~mm}$, where the arrows show direction and magnitude of the velocity. This indicates that phased array vector flow imaging is possible with the approach.

\section{NEW POSSIBILITIES WITH VECTOR VELOCITY ESTIMATION}

The TO approach has now been demonstrated for the conventional 1D transducer geometries and imaging methods, but the approach also opens a whole range of possibilities for new imaging schemes. This includes three dimensional vector velocity estimation, tissue motion and elasticity imaging, transverse spectral velocity estimation, and the derivation of pressure gradients non-invasively. These areas will be briefly described in this Section.

\subsection{Pressure estimation}

An important parameter in characterizing valvular defects and stenosis is the measurement of pressure gradients across the constriction or valve. This is clinically performed by guiding a catheter using X-rays to the site of investigation. ${ }^{25,26}$ This is an invasive procedure with risk for puncture of vessels, bleeding and exposure to ionizing radiation. A non-invasive, fast, and less risky method for pressure gradient measurements would therefore be of great clinical benefit.

The pressure gradients can be determined from the Navier-Stokes equation:

$$
\rho\left[\frac{\partial \vec{v}}{\partial t}+\vec{v} \cdot \nabla \vec{v}\right]=-\nabla p+\rho \vec{g}+\mu \nabla^{2} \vec{v},
$$

where $\rho$ is the density of the fluid, $\vec{v}$ the blood velocity, and $\mu$ its viscosity. The equation relates the forces acting on an isotropic fluid volume to its acceleration and density. The right-hand side sums up the surface and volume forces, which include a pressure term $-\nabla p$, a gravitational term $\rho \vec{g}$ and a term caused by viscous drag. The acceleration term in the bracket on the left-hand side is the substantial derivative* of $\vec{v}^{27}$ Gravitational forces

\footnotetext{
${ }^{*}$ The substantial derivative represents a frame of reference that moves along the particle. It therefore changes as both a function of time and space: $\frac{D}{D t}=\frac{\partial}{\partial t}+\vec{v} \cdot \nabla$
} 

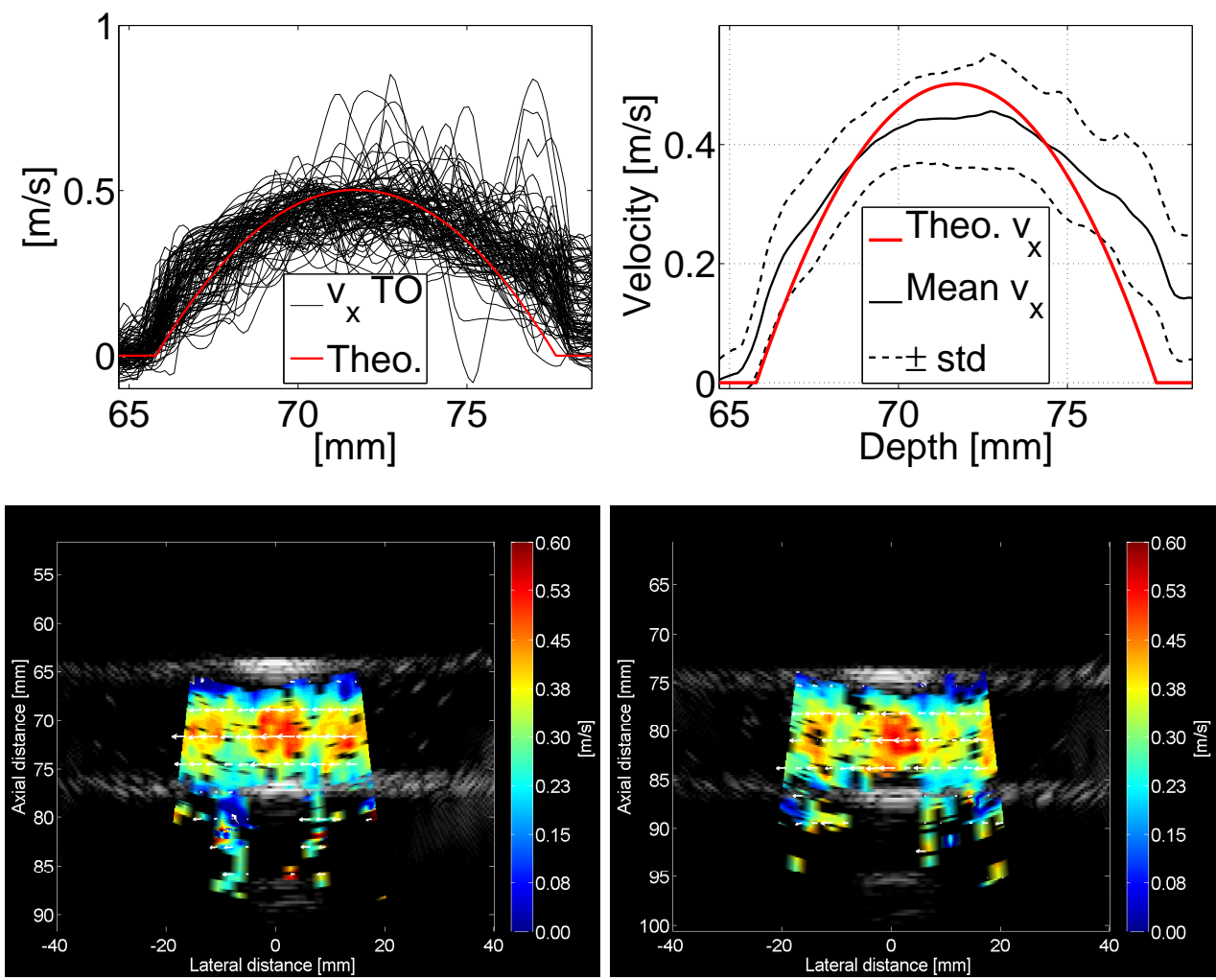

Figure 7. Performance of phased array TO vector velocity estimation. The top graphs shows the individual profiles estimated (left) and the mean \pm one std (right). The two images below show the resulting vector velocity images at 72 and $82 \mathrm{~mm}$, where the arrows show direction and magnitude of the velocity. (From Marcher et al. ${ }^{24}$ ).

can be neglected, if the patient is lying down. The pressure drops due to viscosity can be neglected assuming that the measurement is taking place in a large vessel. Then the pressure gradient is linked solely to the acceleration field and can be calculated from:

$$
\nabla p=-\rho\left[\frac{\partial \vec{v}}{\partial t}+\vec{v} \cdot \nabla \vec{v}\right]
$$

Further assuming that the measurement is performed, so that the out of plane motion is negligible, the pressure gradient can be directly calculated from the vector velocity field found using the TO approach using approximated derivatives like:

$$
\frac{d v}{d x} \simeq \frac{v(x-\Delta x)-v(x+\Delta x)}{2 \Delta x}
$$

An illustration of this is shown in Fig. 8. Here the vector velocity field was measured with a BK Medical ProFocus scanner connected to a research interface, so the RF data could be stored on a PC. The vector velocities were then estimated and the pressure gradients calculated from these. The left graph shows the estimated pressure gradients in the phantom and the right graph shows the corresponding simulated gradients from a Comsol fluid model based on an MRI scan of the phantom (from Olesen et al. ${ }^{28}$ ).

The flow is stationary here and the pressure gradients are principally due to the change in geometry of the vessel. The scanner can, however, measure 20 - 40 frames per second and this opens the possibility for also including the temporal pressure gradient and devise a system for non-invasive and dynamic measurements of pressure gradients. 

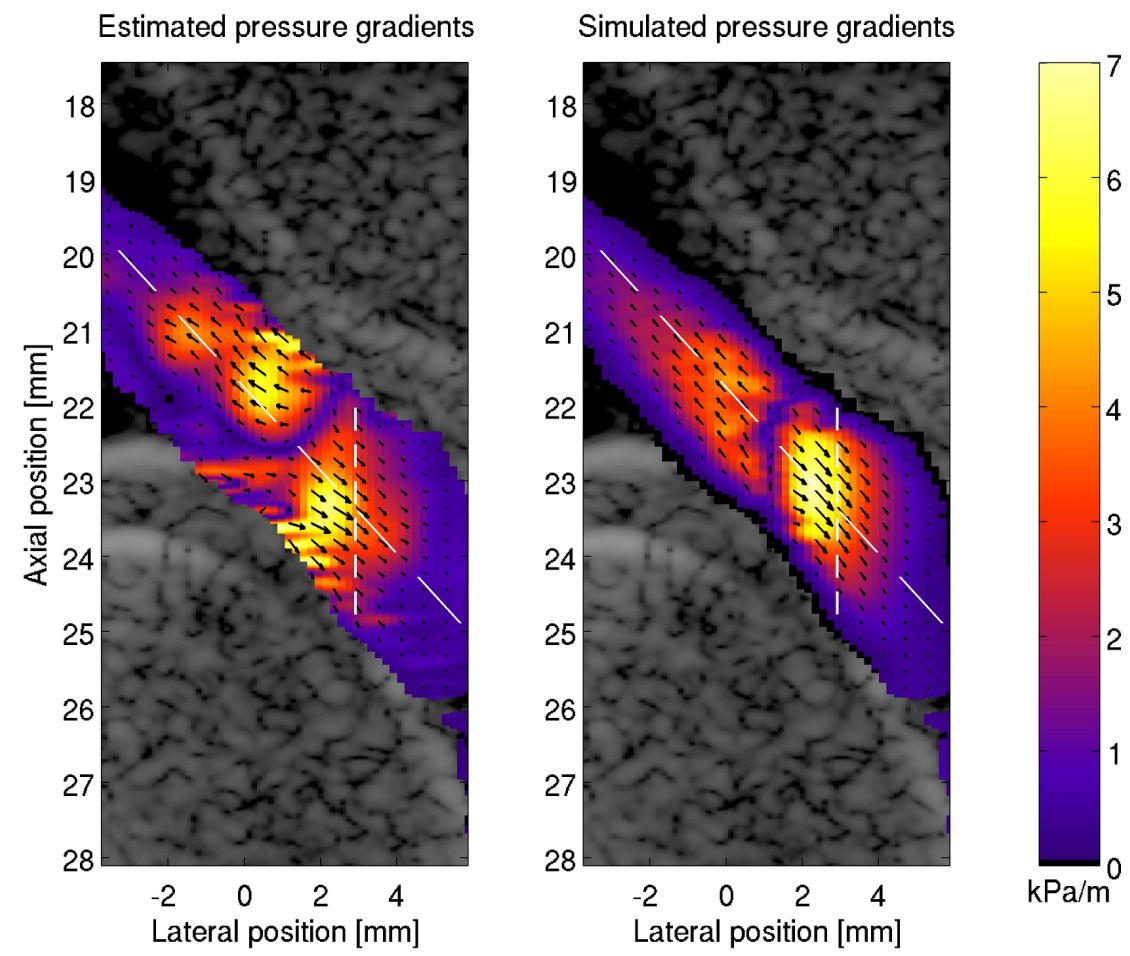

Figure 8. Estimated pressure gradients from a carotid bifurcation phantom (left) and the corresponding simulated results (right). The arrows indicates direction and magnitude of the pressure gradients and the color indicates the magnitude (from Olesen et al. ${ }^{28}$ ).

\subsection{Tissue motion and elasticity estimation}

Tissue motion estimation and elasticity is increasingly getting important in clinical imaging. ${ }^{29,30}$ Here the motion of the tissue is found and from this the elasticity is estimated. ${ }^{31,32}$ The TO approach can also be employed for tissue motion analysis in any direction, if stationary echo canceling is not employed. ${ }^{33}$ This makes it possible also to calculate the elasticity for the two directions.

\subsection{Spectral velocity estimation}

Traditional ultrasound scanners can display the velocity distribution at one given depth in a vessel as a function of time as exemplified by the spectrogram display shown in Fig. 9. The spectrogram is calculated by measuring the sampled signal at the given depth and then employing a short time Fourier transform on the received data. ${ }^{6,34,35}$ The spectra are then stacked side-by-side to show the time evolution of the velocity distribution.

The relation between the blood velocity and the frequency measured $f_{p}$ is given by:

$$
f_{p}=\frac{2 v_{z}}{c} f_{0}=\frac{2|\vec{v}| \cos \Theta}{c} f_{0}
$$

where $v_{z}$ is the blood velocity in the axial direction. $\Theta$ is the angle between the blood velocity vector and the ultrasound beam, and only the projected velocity component is measured by these systems. The measurement should be corrected for this angle, and at 90 degrees no velocity can be found. This is a major problem, since the vessels often are perpendicular to the ultrasound beam direction.

This Section describes a new method for making spectral velocity estimation in the direction transverse to the ultrasound beam based on the TO method. Two different estimation methods are described, and results are shown from their use on simulated data from the femoral artery. 


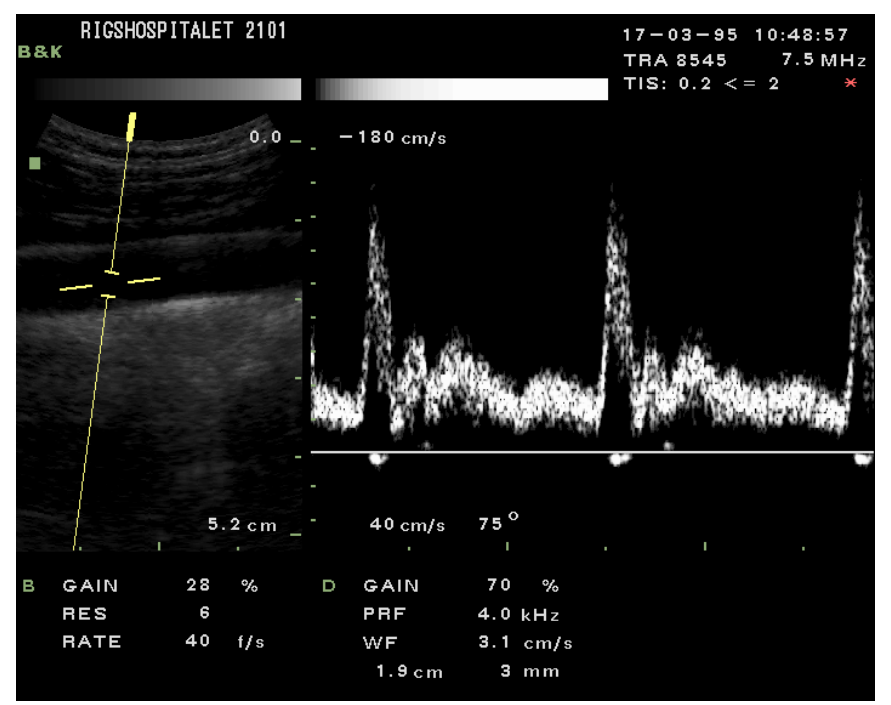

Figure 9. Display of the velocity distribution as a function of time in the carotid artery. The yellow line indicates the place for acquiring the data for the spectrogram.

\subsubsection{Spectral velocity estimator}

The transverse oscillation can be employed in finding the spectrum in the transverse direction with a dedicated estimator. The power density spectrum of a stochastic signal is found from

$$
P_{11}(f)=\sum_{k=-\infty}^{+\infty} R_{11}(k) \exp (-j 2 \pi f k)
$$

where $R_{11}(k)$ is the autocorrelation of the received signal. For the transverse velocity component this can be derived from the cross-correlation function $R_{12}(k)$ between the spatial in-phase and quadrature signal given as

$$
\begin{aligned}
R_{12}(k) & =\sum_{i=-\infty}^{+\infty} r_{1}(i) r_{2}(i+k)=\sum_{i=-\infty}^{+\infty} \exp \left(j 2 \pi i T_{p r f}\left(f_{x}+f_{p}\right)\right) \exp \left(j 2 \pi(i+k) T_{p r f}\left(f_{x}-f_{p}\right)\right) \\
& =\exp \left(j 2 \pi k T_{p r f}\left(f_{x}-f_{p}\right)\right) \sum_{i=-\infty}^{+\infty} \exp \left(j 2 \pi i T_{p r f}\left(f_{x}+f_{p}+f_{x}-f_{p}\right)\right) \\
& =\exp \left(-j 2 \pi k T_{p r f} f_{p}\right) \sum_{i=-\infty}^{+\infty} \exp \left(j 2 \pi i T_{p r f} f_{x}\right) \exp \left(j 2 \pi(i+k) T_{p r f} f_{x}\right)=\exp \left(-j 2 \pi k T_{p r f} f_{p}\right) R_{11}(k)
\end{aligned}
$$

The frequency $f_{p}=\frac{2 v_{z}}{c} f_{0}$ is zero in the case there is no axial velocity component $\left(v_{z}=0\right)$ and the crosscorrelation directly equals the autocorrelation of the transverse signal. Making a short Fourier transform of this, thus, directly reveals the transverse velocity spectrum. This is called the second order approach.

The modulation of the cross-correlation function by the factor $\exp \left(-j 2 \pi k T_{p r f} f_{p}\right)$ can be compensated for by estimating the axial velocity and thereby $f_{p}$, and then multiply $R_{12}(k)$ by the compensation factor

$$
R_{c}(k)=\exp \left(j 2 \pi k T_{p r f} f_{p}\right) .
$$

The axial velocity can be found from a normally focused line lying between the two spatial beams and then employ the autocorrelation estimator given by: ${ }^{6,7}$

$$
\hat{v}_{z}=-\frac{c f_{p r f}}{4 \pi f_{0}} \arctan \left(\frac{\sum_{i=1}^{N_{c}-1} y(i) x(i-1)-x(i) y(i-1)}{\sum_{i=1}^{N_{c}-1} x(i) x(i-1)+y(i) y(i-1)}\right) .
$$


where the received $\mathrm{RF}$ signal is $r(i)=x(i)+j y(i)$.

The compensation for the axial velocity can also be made using a fourth order estimator similar to the one derived in. ${ }^{12}$ The fourth order correlation function is calculated as:

$$
R_{11}(k)=\sum_{i=-\infty}^{+\infty} r_{1}(i) r_{1}(i+k), \quad R_{22}(k)=\sum_{i=-\infty}^{+\infty} r_{2}(i) r_{2}(i+k), \quad R_{44}(k)=R_{11}(k) \cdot R_{22}(k),
$$

which eliminates the axial component. An elimination of the lateral component can be done using

$$
R_{44 a x}(k)=R_{11}(k) \cdot R_{22}^{*}(k) .
$$

The spectrum is then found from:

$$
P_{44}(f)=\sum_{k=-\infty}^{+\infty} R_{44}(k) \exp (-j 2 \pi f k)
$$

This approach is called the fourth order spectral estimation method.

\subsubsection{Simulation of angle independent spectral estimation}

Blood flow in the femoral artery has been simulated using Field II and the Womersley-Evan's pulsatile flow model. ${ }^{36}$ The model gives realistic simulated velocity profiles for a normal human geometry. The parameters for the transducer given in Table 1 are used in the simulation to yield the TO fields. The electronic focus in transmit is now $200 \mathrm{~mm}$ and the $\mathrm{F} \#$ in transmit is 5 .

The resulting power density spectrograms for different angles for the second order method are shown in the left column of Fig. 10. The velocity distribution is reliably estimated at $90^{\circ}$, but gets progressively worse for angles different from $90^{\circ}$. The same spectra for the fourth order methods are shown in the right column of Fig. 10. It can be seen that the spectrogram can be estimated for a fully transverse flow. The blue line indicates the correct mean velocity in the spectrum. The estimates get progressively worse when the beam to flow angle deviates from 90 degrees, but this is not a major problem. For angles less than 60 degrees the normal spectrogram can be used and gives a good estimate. The angle can easily be estimated from the TO data measured for the spectral estimation and be used for selecting which spectrum to display.

\subsection{Three-dimensional vector velocity imaging}

The full three-dimensional velocity vector should be estimated as a function of time and space to capture the full extent of blood's motion in the human circulation. This necessitates the use of a two-dimensional array and an adaptation of the approach to also estimate the out-of-plane velocity. It is important that all three components are estimated simultaneously as both magnitude and direction rapidly changes during the cardiac cycle.

This can be accomplished by using a $32 \times 32$ elements phased array matrix probe and sending out a weakly focused field. ${ }^{37,38}$ Five lines are then beamformed in parallel during receive processing to generate signals for finding the axial velocity component $v_{z}$, the lateral velocity component $v_{x}$, and the elevation component $v_{y}$. For the last two components the TO approach is used, and in-phase and quadrature beams are focused in the lateral direction for finding $v_{x}$ together with the in-phase and quadrature beams in the elevation direction for finding $v_{y}$. The beams are generated by making a dual peak apodization on the receiving aperture as shown in white in Fig. 11 along the direction of the velocity estimation and focusing as described for the phased array probe for generating the in-phase and the quadrature beams. The TO velocity estimation is then employed to yield the velocity components.

This approach has been implemented on the SARUS experimental scanner ${ }^{22}$ using a $3.5 \mathrm{MHz}$ matrix probe with $32 \times 32$ elements fabricated by Vermon S.A. (Tours, France). ${ }^{40}$ A weakly focused ultrasound field is emitted and the RF signals from all 1024 elements are stored in memory and then beamformed off-line. ${ }^{38}$ Data has been acquired from stationary parabolic flow in a flow rig, and the resulting profiles are shown in Fig. 12 for all three velocity components for simulated data (left columns) and measured data (right columns). The left column shows the results for a motion in the $x$ direction only and the right for the $y$ direction only. It can be seen that the estimators can find the correct velocity components and thereby the full velocity vector. 

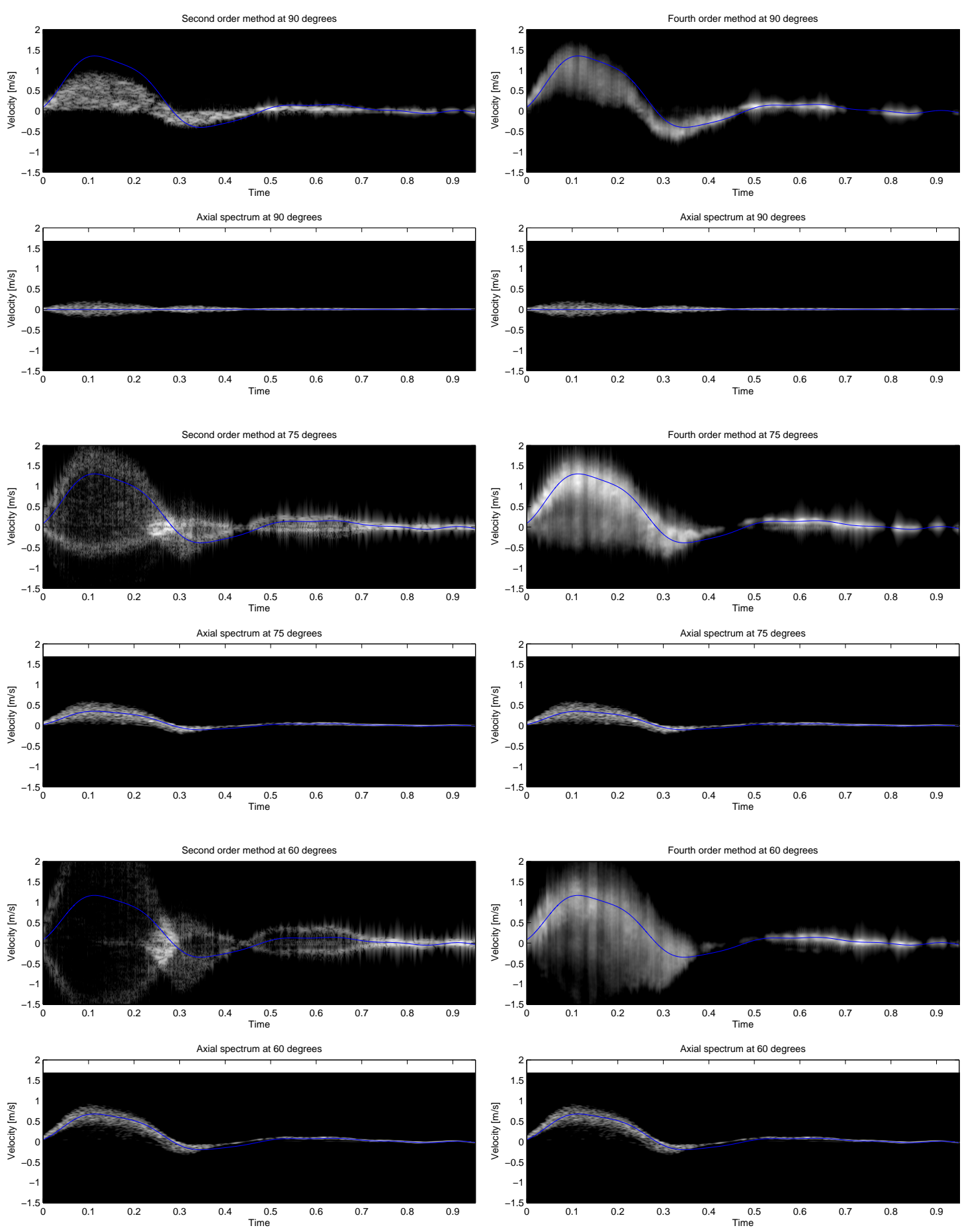

Figure 10. Estimated power density spectrograms for the second order (left) and fourth order methods (right) for simulated flow in the femoral artery. Pairs of spectra as shown with the lateral velocity spectrum shown on the top image and the axial velocity spectrum at the bottom for three different angles at $90^{\circ}$ (top two graphs), $75^{\circ}$ (middle) and $60^{\circ}$ (bottom). The blue line indicates the correct mean velocity in the spectrum. 

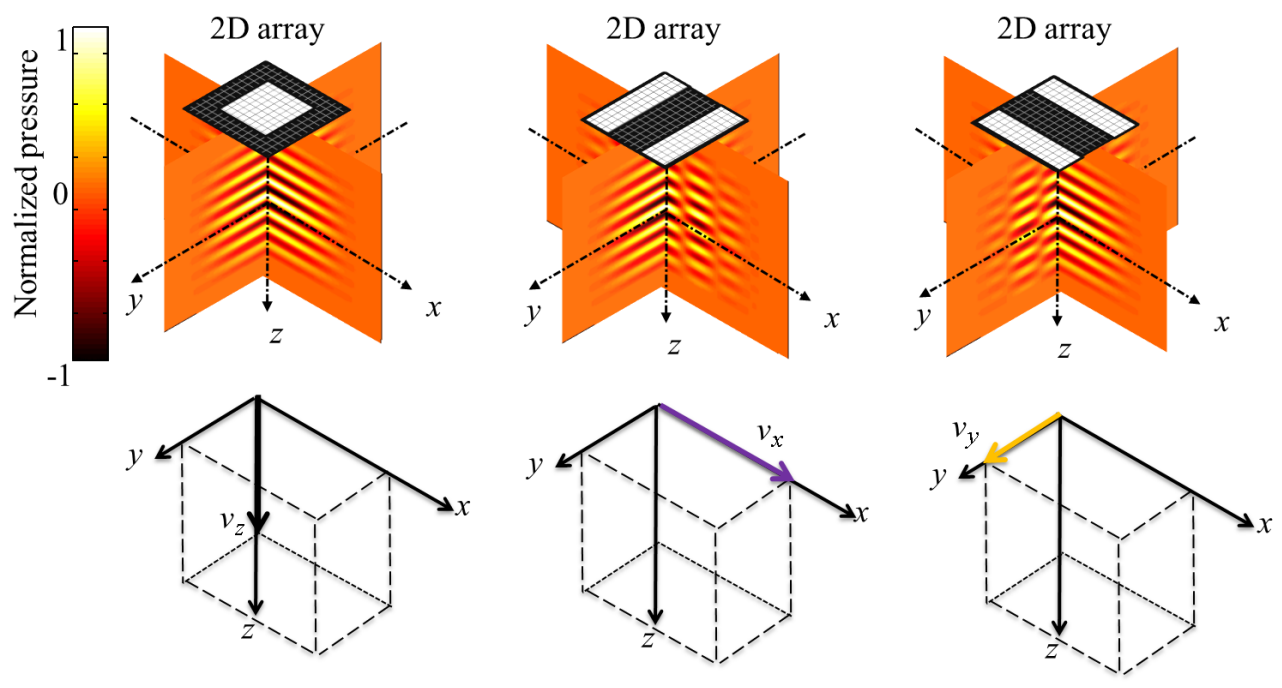

Figure 11. Apodization used during receive processing for the three different velocity components. The white areas of the transducer denotes the active elements during receive focusing (from Pihl et al. ${ }^{39}$ ).
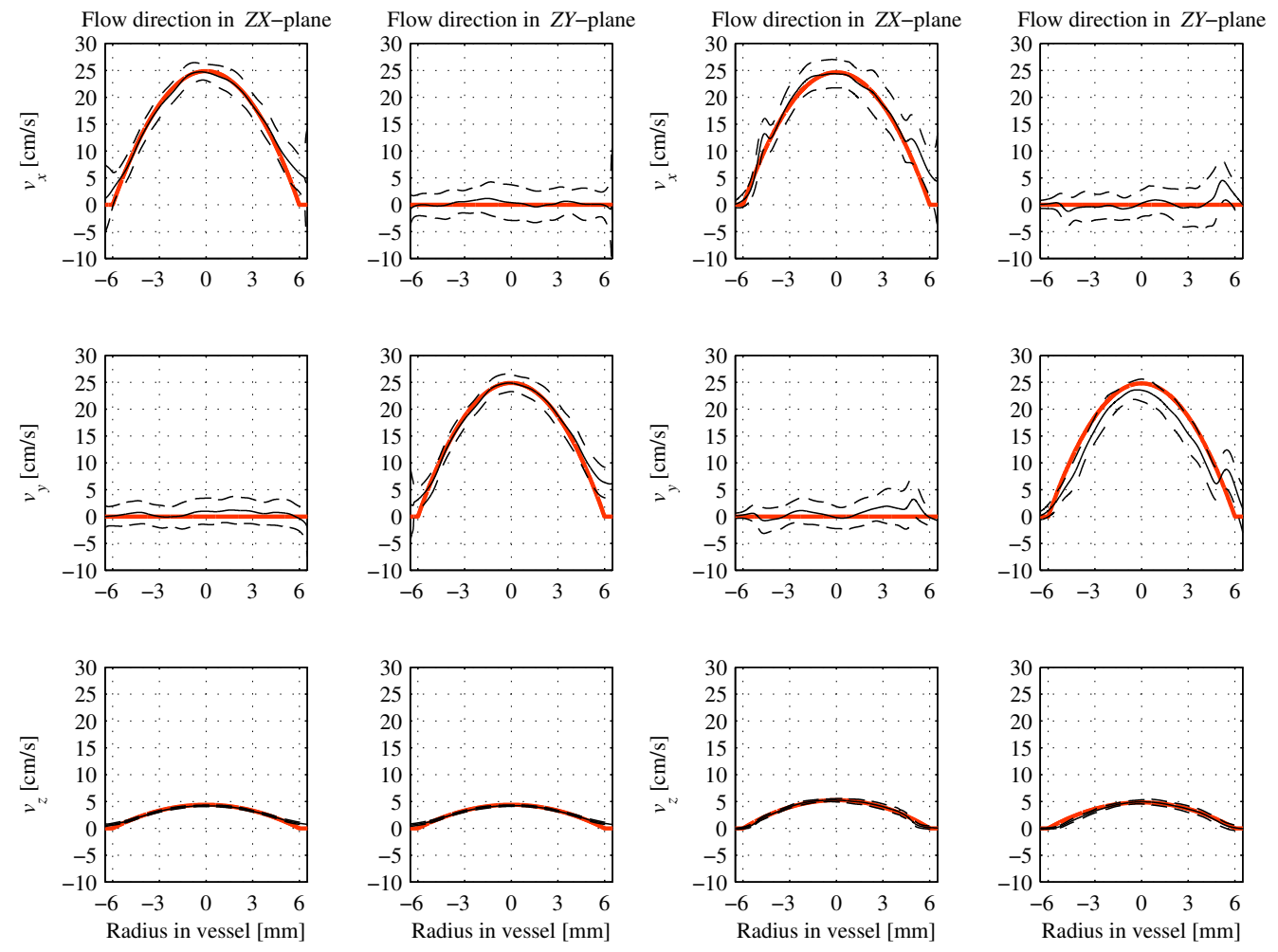

Figure 12. Mean and standard deviation of 50 measured velocity profiles for the three estimated velocity components. The left figures are simulated and the right figures are the measured profiles. Thick red lines indicate the expected velocity profile, thin lines indicate the mean of the profiles, and dashed lines one standard deviation. The actual beam-to-flow angles were $78^{\circ}$ and $79^{\circ}$, respectively (from Pihl et al. ${ }^{38}$ ). 

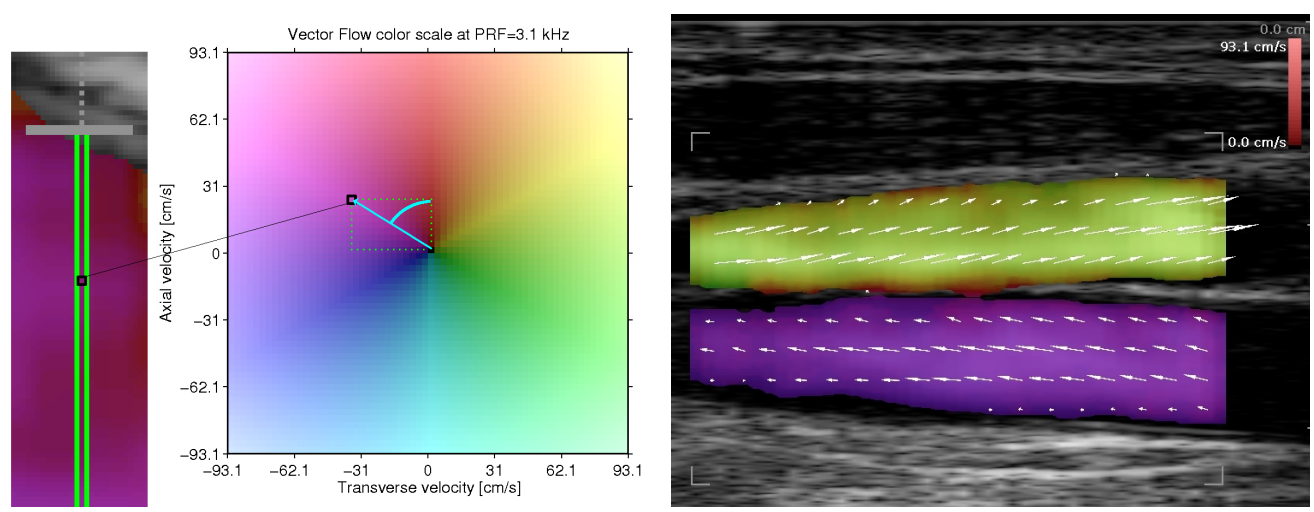

Figure 13. The left figure shows the color encoding used in the BK Medical ProFocus scanner for displaying the vector velocity. The color indicates direction and the intensity magnitude. The right image shows an example from the internal jugular vein and common carotid artery at a nearly 90 degrees scan angle. The arrows also indicate direction and magnitude.

\section{CLINICAL EXAMPLES OF VECTOR VELOCITY ESTIMATION}

The TO approach for vector velocity imaging is implemented on the BK Medical ProFocus platform (BK Medical, Herlev, Denmark) and is now FDA approved and employed in the clinic. It has been validated against MRI phased-contrast images and shown to yield the same stroke volume in the carotid artery as the MR scans with a correlation coefficient of $0.91 .^{18}$ Examples of its use has been shown in several papers, ${ }^{3,4,41,42}$ and a few examples are shown below.

The color encoding used by the BK Medical ProFocus scanner is shown on the left in Fig. 13, where the color indicates direction and the intensity magnitude. The superimposed arrows also indicate direction and magnitude. The right image shows an example from the internal jugular vein and common carotid artery at a nearly 90 degrees scan angle, where other scanners would show zero or very low velocities. It is here seen that the velocities have opposite directions and approaches the correct magnitude in these types of vessels. ${ }^{18}$ This shows that the correct velocity magnitude is attained irregardless of the transducer placement, and it is not necessary to angle the ultrasound imaging direction or correct for the flow angle. This can potentially give an improvement of the clinical work flow.

Fig. 14 shows flow through a valve in the femoral vein. The left image shows the open valve with flow through and vortices forming behind the valve leaflets. The zoomed right image shows that the closing mechanism of the valve is incomplete and retrograde flow is seen on the right image between the cusps, resulting in venous insufficiency. The femoral artery is seen parallel to the vein. The flow depicted here is much more complicated and dynamic than in straight vessels. Both magnitude and direction changes at any position in the vessel and over time. Here it is, thus, not possible to correct with a single flow angle as it varies spatially over time.

The left image of Fig. 15 shows an arteriovenous fistula (surgical joining a vein and an artery) for use in hemodialysis. The recording is centered in the anastomosis in which the arterial blood from the bottom left (yellow pixels) must pass partly to the return vein and partly to the artery on the right. The anastomosis is not made correctly, and there are pronounced turbulence with very limited flow towards the right in the artery. An other example of a stenosed fistula is shown on the right.

Scanning of the heart during open chest surgery has been performed for the images shown in Fig. 16. The left image shows a systolic jet formed between the aortic cusps at the bottom left corner. An antegrade flow centrally in the ascending aorta is seen with retrograde flow along the vessel walls and vortices in the aortic sinuses during systole. The right image shows a clock-wise secondary flow during peak systole in the ascending aorta. The image shows that the TO method can reveal complex flow in any direction dynamically with 20 frames per second. It clearly indicates both the direction and the magnitude of the flow and makes it possible to study valve defects, vortices, rotational flow and other complex hemodynamics. 

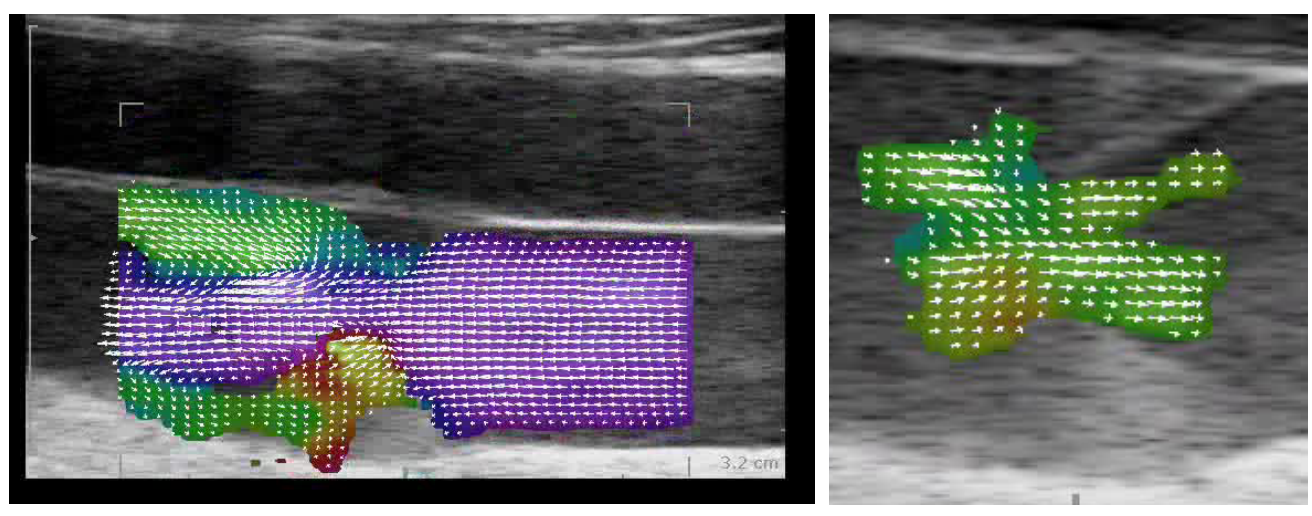

Figure 14. Scan of the femoral vein at the level of a venous valve. Notice the antegrade jet between the cusps and the well-defined vortices down-stream of the valve on the left image. The closing mechanism of the valve is incomplete and retrograde flow is seen on the right image between the cusps, resulting in venous insufficiency. The femoral artery is seen parallel to the vein.
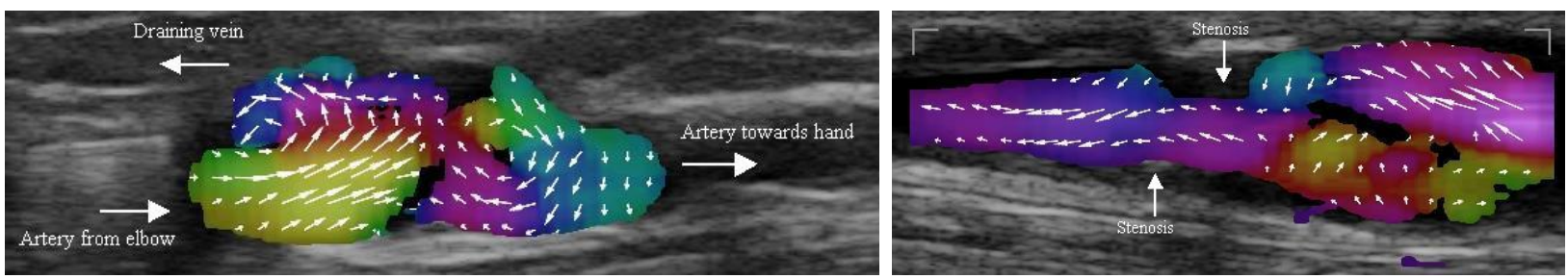

Figure 15. The left scan shows an arteriovenous fistula. It is a surgically created connection between an artery and a vein in the forearm and is used for hemodialysis treatment. The connection is not made properly, and too much blood passes from the artery to the vein, leaving the rest of the forearm and hand with insufficient blood supply. Pronounced turbulence is formed in the connection, and very little blood continues in the artery toward the hand. The right scan shows the draining vein of a surgically created arteriovenous fistula. The vein has a stenosis, which decreases the blood flow and creates turbulence.
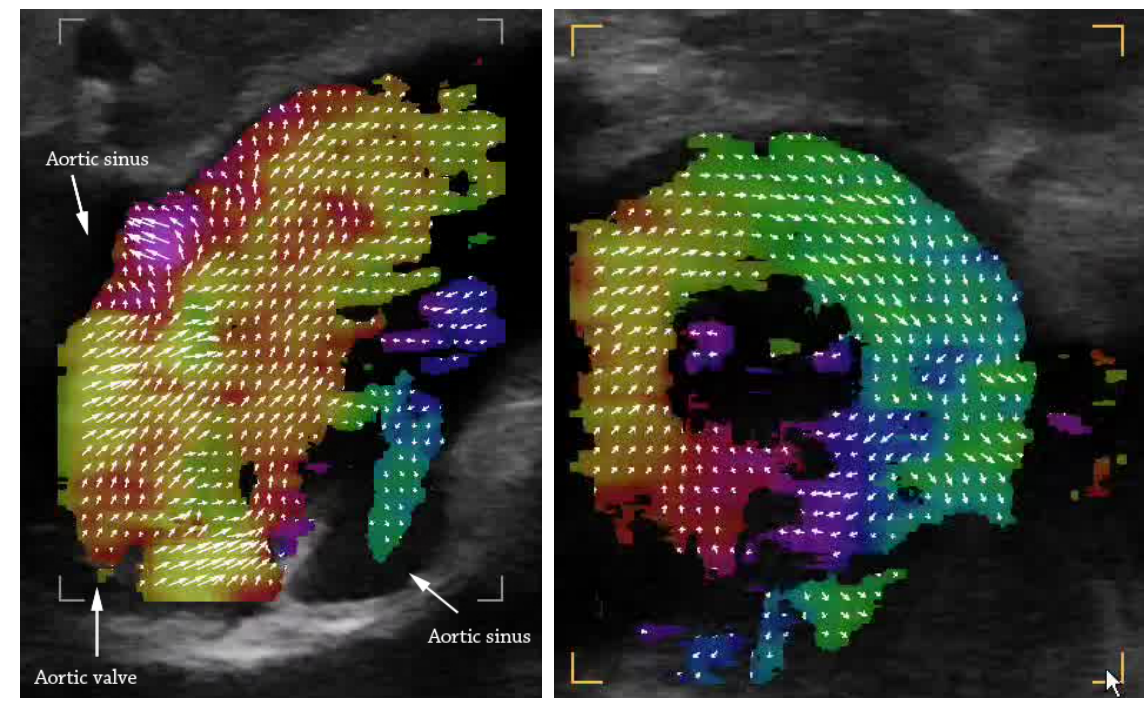

Figure 16. The left image shows a systolic jet formed between the aortic cusps (bottom left corner). An antegrade flow centrally in the ascending aorta is seen with retrograde flow along the vessel walls and vortices in the aortic sinuses during systole. The right image shows a clock-wise secondary flow pattern during peak systole in the ascending aorta. 


\section{SUMMARY}

Blood velocity visualization can easily and conveniently be performed with medical ultrasound, but the estimated velocity is only the component along the ultrasound beam. Traditional scanners can make angle correction by tilting the image direction and setting the angle. This is usable for quantitative analysis when a laminar flow with a stable direction is found, but this is far from correct in the human circulation. Several clinical examples have been shown demonstrating that pulsatile flow changes direction over the cardiac cycle as a function of spatial position. A correct compensation must therefore be performed for any time and position in the image, which obviously is impossible in a clinical setting. The TO vector velocity can automatically reveal the correct velocity vector and dynamically reveal the pulsatility and directional changes in the human circulation. This is encountered in nearly any site in the vessels apart from the simplest of cases. The method has been commercially implemented and FDA approved and a number of clinical examples have demonstrated the use of the technique for linear array scanning in the carotid artery, peripheral vessels, and the heart.

The new method is implemented on a portable platform and reveals the velocity vector in real time making it possible to perform several measurements and manipulate the region under investigation. Interaction in the operating theater during e.g. cardiac surgery is also possible. This is a new addition to vector velocity imaging, which traditionally had to be performed using MR scanning and acquisitions for several hundred ECG gate cardiac cycles. This measurement is also performed without direct access to the patient making it difficult, if not impossible, to make dynamic studies with patient interaction.

It has also been demonstrated that the TO method can be applied to other transducer geometries. This includes phased arrays for cardiac imaging and convex arrays for abdominal scanning. The ultrasound fields are highly complex due to the lateral oscillation and computer optimization is needed to get a 90 degrees phase shift between the in-phase and quadrature beams. Such optimization also reduces both bias and standard deviation of the final estimates.

Introducing 2D arrays makes it possible to expand the method to full three-dimensional vector velocity imaging, where also the out-of-plane component is estimated simultaneously with the two other components. This makes it possible to perform velocity tensor imaging, where the $3 \mathrm{D}$ velocity tensor is found as function of the three spatial coordinates and time.

A new approach for angle independent spectral velocity estimation has been presented in both a second order and a fourth order version. Both estimators can find the spectrum at 90 degrees, where a traditional estimator yields a zero velocity. The spectral estimates degrade when the angle is different from 90 degrees. It degrades more quickly for the second order approach than for the fourth order approach, which works up to 70-60 degrees. After this the traditional spectrum estimate is best and should be used. The selection between the two spectra can be based on the estimated angle at the range gate, which can be obtained using the normal TO estimator without additional beamforming. It is should be noted that the velocity range is significantly higher for the new fourth order method compared to traditional estimation, as the lateral wavelength is large than the axial wavelength. That is beneficial for either keeping the pulse repetition frequency low or for maintaining a high maximum detectable velocity.

The data from the TO method can also be employed in other estimation procedures. This includes using the Navier-Stokes equation for deriving the pressure gradients in the vessels across valves and stenosis. This can potentially substitute or even replace catherization to find the internal pressure gradients. Another possible application is in tissue motion and elasticity imaging to e.g. reveal properties of the vessel walls and the heart muscle.

\section{ACKNOWLEDGMENTS}

This work was supported by grant 9700883, 9700563 and 26-04-0024 from the Danish Science Foundation and by B-K Medical, Herlev, Denmark along with support by grant 024-2008-3 from the Danish Advanced Technology Foundation. The authors would also like to thank Peter Munk, Jesper Udesen, Mads Møller Pedersen, Per Haugaard and Jønne Marcher for their many contributions to the development and investigation of TO imaging. 


\section{REFERENCES}

[1] Van Der Geest, R., Niezen, R., Van Der Wall, E., De Roos, A., and Reiber, J., "Automated measurement of volume flow in the ascending aorta using MR velocity maps: Evaluation of inter- and intraobserver variability in healthy volunteers," J. Comput. Assist. Tomogr. 22(6), 904-911 (1998).

[2] Spilt, A., Frieke, M., Van Der Geest, R., Reiber, J., Kunz, P., Kamper, A., Blauw, G., and Van Buchem, M., "Reproducibility of total cerebral blood flow measurements using phase contrast magnetic resonance imaging," J. Magn. Reson. Imaging, 16(1), 1-5 (2002).

[3] Evans, D. H., Jensen, J. A., and Nielsen, M. B., "Ultrasonic colour Doppler imaging," Interface Focus 1, 490-502 (August 2011).

[4] Hansen, P. M., Pedersen, M. M., Hansen, K. L., Nielsen, M. B., and Jensen, J. A., "Demonstration of a vector velocity technique," Ultraschall in Med 32, 213-5 (2011).

[5] Evans, D. H. and McDicken, W. N., [Doppler Ultrasound, Physics, Instrumentation, and signal processing], John Wiley \& Sons, New York (2000).

[6] Jensen, J. A., [Estimation of Blood Velocities Using Ultrasound: A Signal Processing Approach], Cambridge University Press, New York (1996).

[7] Kasai, C., Namekawa, K., Koyano, A., and Omoto, R., "Real-Time Two-Dimensional Blood Flow Imaging using an Autocorrelation Technique," IEEE Trans. Son. Ultrason. 32, 458-463 (1985).

[8] Loupas, T., Powers, J. T., and Gill, R. W., "An axial velocity estimator for ultrasound blood flow imaging, based on a full evaluation of the Doppler equation by means of a two-dimensional autocorrelation approach," IEEE Trans. Ultrason., Ferroelec., Freq. Contr. 42, 672-688 (1995).

[9] Munk, P., Estimation of the 2-D flow vector in ultrasonic imaging: a new approach, Master's thesis, Department of Information Technology, Technical University of Denmark (1996).

[10] Jensen, J. A. and Munk, P., "A New Method for Estimation of Velocity Vectors," IEEE Trans. Ultrason., Ferroelec., Freq. Contr. 45, 837-851 (1998).

[11] Anderson, M. E., "Multi-dimensional velocity estimation with ultrasound using spatial quadrature," IEEE Trans. Ultrason., Ferroelec., Freq. Contr. 45, 852-861 (1998).

[12] Jensen, J. A., "A New Estimator for Vector Velocity Estimation," IEEE Trans. Ultrason., Ferroelec., Freq. Contr. 48(4), 886-894 (2001).

[13] Bracewell, R., [The Fourier transform and its applications], McGraw-Hill, New York, 3rd ed. (1999).

[14] Jensen, J. A. and Svendsen, N. B., "Calculation of Pressure Fields from Arbitrarily Shaped, Apodized, and Excited Ultrasound Transducers," IEEE Trans. Ultrason., Ferroelec., Freq. Contr. 39, 262-267 (1992).

[15] Jensen, J. A., "Field: A program for simulating ultrasound systems," Med. Biol. Eng. Comp. 10th NordicBaltic Conference on Biomedical Imaging, Vol. 4, Supplement 1, Part 1, 351-353 (1996).

[16] Pihl, M. J., Marcher, J., and Jensen, J. A., "Phased-array vector velocity estimation using transverse oscillations," IEEE Trans. Ultrason., Ferroelec., Freq. Contr. 59, 2662-2675 (December 2012).

[17] Udesen, J. and Jensen, J. A., "Investigation of Transverse Oscillation Method," IEEE Trans. Ultrason., Ferroelec., Freq. Contr. 53, 959-971 (2006).

[18] Hansen, K. L., Udesen, J., Thomsen, C., Jensen, J. A., and Nielsen, M. B., "In vivo validation of a blood vector velocity estimator with MR angiography," IEEE Trans. Ultrason., Ferroelec., Freq. Contr. 56(1), 91-100 (2009).

[19] Pihl, M. J., 3D vector flow imaging, PhD thesis, Technical University of Denmark (2012).

[20] Marcher, J., Vector Velocity Imaging using a Commercial Scanner - Implementation of the Transverse Oscillation Method using a Phased Array Transducer, Master's thesis, Center for Fast Ultrasound Imaging, Department of Electrical Engineering, Technical University of Denmark, Lyngby, Denmark (March 2012).

[21] Liebgott, H., Basarab, A., Marincas, S., Bernard, O., and Friboulet, D., "Tangential oscillations for motion estimation in echocardiography," in [Proc. IEEE Ultrason. Symp.], $1761-1764$ (nov. 2008).

[22] Jensen, J. A., Holten-Lund, H., Nielson, R. T., Tomov, B. G., Stuart, M. B., Nikolov, S. I., Hansen, M., and Larsen, U. D., "Performance of SARUS: A Synthetic Aperture Real-time Ultrasound System," in [Proc. IEEE Ultrason. Symp.], 305-309 (Oct. 2010). 
[23] Hemmsen, M. C., Nikolov, S. I., Pedersen, M. M., Pihl, M. J., Enevoldsen, M. S., Hansen, J. M., and Jensen, J. A., "Implementation of a versatile research data acquisition system using a commercially available medical ultrasound scanner," IEEE Trans. Ultrason., Ferroelec., Freq. Contr. 59(7), 1487-1499 (2011).

[24] Marcher, J., Pihl, M. J., and Jensen, J. A., "The transverse oscillation method using a phased array transducer," in [Proc. IEEE Ultrason. Symp.], Accepted (2012).

[25] Baim, D. S. and Grossman, W., [Grossman's cardiac catheterization, angiography, and intervention], Lippincott Williams \& Wilkins publishers (2000).

[26] Skinner, J. S. and Adams, P. C., "Outpatient cardiac catheterisation," Int. J. Cardiol. 53, 209-219 (March 1996).

[27] Truskey, G. A., Yuan, F., and Katz, D. F., [Transport Phenomena in Biological Systems], Pearson Education, Inc., Pearson Prentice Hall (2004).

[28] Olesen, J. B., Traberg, M. S., Pihl, M. J., and Jensen, J. A., "Non-invasive measurement of pressure gradients using ultrasound," in [Proc. SPIE Med. Imag.], Accepted (March 2013).

[29] Barbone, P. and Bamber, J., "Quantitative elasticity imaging: What can and cannot be inferred from strain images," Phys. Med. and Bio. 47(12), 2147-2164 (2002).

[30] Greenleaf, J., Fatemi, M., and Insana, M., "Selected methods for imaging elastic properties of biological tissues," Ann. Rev. Biomed. Eng. 5, 57-78 (2003).

[31] Konofagou, E. and Ophir, J., "A new elastographic method for estimation and imaging of lateral displacements, lateral strains, corrected axial strains and poisson's ratios in tissues," Ultrasound Med. Biol. 24(8), 1183-1199 (1998).

[32] Yamakoshi, Y., Sato, J., and Sato, T., "Ultrasonic imaging of internal vibration of soft tissue under forced vibration," IEEE Trans. Ultrason., Ferroelec., Freq. Contr. 37(2), 45-53 (1990).

[33] Liebgott, H., Wilhjelm, J., Jensen, J. A., Vray, D., and Delachartre, P., "PSF dedicated to estimation of displacement vectors for tissue elasticity imaging with ultrasound," IEEE Trans. Ultrason., Ferroelec., Freq. Contr. 54(4), 746-756 (2007).

[34] Baker, D. W., "Pulsed ultrasonic Doppler blood-flow sensing," IEEE Trans. Son. Ultrason. SU-17, 170-185 (1970).

[35] Evans, D. H., McDicken, W. N., Skidmore, R., and Woodcock, J. P., [Doppler Ultrasound, Physics, Instrumentation, and Clinical Applications], John Wiley \& Sons, New York (1989).

[36] Evans, D. H., "Some aspects of the relationship between instantaneous volumetric blood flow and continuous wave Doppler ultrasound recordings III," Ultrasound Med. Biol. 8, 617-623 (1982b).

[37] Pihl, M. J. and Jensen, J. A., "3D velocity estimation using a 2D phased array," in [Proc. IEEE Ultrason. Symp.], 430-433 (2011).

[38] Pihl, M. J. and Jensen, J. A., "Measuring 3D velocity vectors using the transverse oscillation method," in [Proc. IEEE Ultrason. Symp.], IEEE (2012).

[39] Pihl, M. J., Stuart, M. B., Tomov, B. G., Hansen, J. M., Rasmussen, M. F., and Jensen, J. A., "Preliminary example of 3D vector flow imaging," in [Proc. SPIE Med. Imag.], Accepted (2013).

[40] Ratsimandresy, L., Mauchamp, P., Dinet, D., Felix, N., and Dufait, R., "A 3 MHz two dimensional array based on piezocomposite for medical imaging," in [Proc. IEEE Ultrason. Symp.], 1265-1268 (2002).

[41] Udesen, J., Nielsen, M. B., Nielsen, K. R., and Jensen, J. A., "Examples of in-vivo blood vector velocity estimation," Ultrasound Med. Biol. 33, 541-548 (2007).

[42] Hansen, K. L., [In-vivo studies of new vector velocity and adaptive spectral estimators in medical ultrasound], vol. 57(5), pp. 1-23, Dan Med Bull. (2010). 